\title{
Meta
}

Journal des traducteurs

Translators' Journal

\section{Principe de la traduction - principe du langage : l'autrement-dit}

\section{Boris Lobatchev}

Volume 40, numéro 4, décembre 1995

URI : https://id.erudit.org/iderudit/003513ar

DOI : https://doi.org/10.7202/003513ar

Aller au sommaire du numéro

Éditeur(s)

Les Presses de l'Université de Montréal

ISSN

0026-0452 (imprimé)

1492-1421 (numérique)

Découvrir la revue

Citer cet article

Lobatchev, B. (1995). Principe de la traduction - principe du langage :

l'autrement-dit. Meta, 40(4), 707-713. https://doi.org/10.7202/003513ar

\section{Résumé de l'article}

Depuis la Tour de Babel, on a pris une fâcheuse habitude de penser les traducteurs comme sauveurs de l'humanité. Aussi involontairement les cite-t-on en exemple dans cette affirmation de Jakobson que dire c'est traduire. Ici, je vais essayer de prouver qu'au contraire traduire ne fait que suivre, et cela jusqu'à partager avec le dernier ce principe selon lequel rien n'est sans " autrement-dit ». Quant à la mission sacrée, ça dépend de l'interprète qui doit apprendre à dire les choses autrement, tout en laissant confondre son dire avec celui des autres 


\section{PAINCIPE DE LA TRADUCTION - PAINCIPE DU LANGAGE : L'AUTREMENT-DIT}

\section{Résume}

Depuis la Tour de Babel. on a pris une facheusp habirude de penser les traducteurs comme sauveurs de l'humanifé. Aussi involontairement les cite-t-on en exemple dans cette affirmation de Jakohson que dire c'est traduire. lii. je lais essaver de prowier qu'au contraire traduire ne fait que suivre suivre, ef cela jusqu'd partager aver le dernier ce principe selon lequel rien n'est sans rautrement-ditw. Quant d la mission sacréé, sa dépend de l'interprèse qui doit apprendre d dire les choses autrement. tout en laissant confondre son dire anec celui des autres.

\section{Abstract}

Ever since the Tower of Babel, translators have unfortunately been considered as saviours of humanity. They are inadvertently singled out in Jakobson's assertion that ranslating is a way of saving things Nevertheless, the author sets out to show that ranslating is but following a given path. and goes on to asree with Jakobson that evervthing (an be rew'orded ("I autrement-dit"). As for the sacred mission. it depends on the interpreter whe mast learn of reword. while adapting his n'as of saving things to others

"La terre est surpeuplec.

D'un peuple a l'autre. il n'y a rien à voir. Nul point commun.

Hors l'horizon.

Il faudrait rassembler tout le monde en un seul point.

Pour retrouver l'humanite."

( "Babel ou le mystère des langues», piece de Michel Pironi et Jacques Roux. Les (ahiers de l'Exare. 1993. pp. 9-10)

\section{INTRODUCTION}

Passant d'une langue a l'autre, tout traducteur cherche à procéder d'une facon la plus organique possible. Or, pour y arriver, il faudrait d'abord realiser le rêve de orassembler tout le monde en un seu point", essayer de faire coïncider le principe de la traduction avec celui des langues différentes, celui du langage humain.

Sans ètre véritablement connu, le principe des langues est communement ressenti depuis Babel. C'est un autrement-dit général. Effectivement, il est difficile, sinon impossible. de ne pas , apercevoir que les langues se parlent differemment les unes des autres.

Logiquement, I'idée me paraît évidente, mas psychologiquement, à cause de son caractère dérangeant. elle n'est pas facile à admettre. La preuve qu'on retoume nostalgiquement au mythe de Babel (voir notre exergue), au temps d" "avant" (et d' «apres") à la confusion des langues (cf. Nouss 1990). La récente tentative (1993) du même auteur de découvrir le langage universel ailleurs que dans la langue en est une autre. Tournée vers la musique qui fait partie de la poésie, elle va aussi dans un sens oppose. celui du proprement-dit. Mais scruter cel idéal au-delà des mots, c'est reconnaitre son absence dans le domaine verbal qui est le nôtre.

Bref, l'idee est dans l'air, et elle ne peut ne pas passer aussi par tout ce qui touche a la traduction. Notons des ouvrages intitules de plus en plus souvent d'une façon significalive: In other nords. Notons également des emplois, encore hésitants, entre guillemets, occasionnels, de "dire autrement" par opposition a adire autre chose", mais toujours en rappon avec le franchissement des frontières linguistiques ( $f$. Gouadec 1990 ): 340).

Naturellement. ce ne sont la que des approches intuitives. Mais il est normal que l'idée de l'autrement-dit soit adoptée d'abord sous sa forme la plus transparente, celle de synonymie interlinguistique : maison, casa, house, Haus, dom, etc

Pour aller plus loin que les équivalences relativement élémentaires, force nous est d'approfondir icı la notion de l'autrement-dit' '. À cette fin. nous allons commencer par le plan intralinguistique - le langage en general - passer ensuite au plan interlinguistique - les langues particulieres - pour finir par les plans winter-intra-linguistiques superposes" .- la traduction.

\section{L'AUTREMENT-DIT :}

\section{PRINCIPE DU LANGAGE}

Prendre nos désirs pour la réalité est tellement humain qu'il n'y a rien d'étonnant à ce qu'on ait tendance à prêter au langage nos intentions. II nous paraît alors logique, equilibré, bien raisonné. N'est-ce pas le danger contre lequel nous prévenait déja Michel Breal (1897: 4)? Pas d'anthropomorphisme excessif !

Eh bien, à regarder notre langage d'un ail detache. comme le faisait, entre autres. Barthes (1984), à examiner. comme nous le faisons, le jeu du 
fond et de la forme. on finit par remarquer ceci : une partic importante de la forme se recoupant avec lo fond, elle demeure inexprimée. Et c'est à partir des éléments donnés que l'auditeur est oblige de la déduire. Par exemple, l'un dit un proverbe incomplet Ricon ne sert de ivurir... et l'autre en reconstituc l'ensemble, ajoutant : il faut partir a poime. Ine autre partic, non moins importante, du fond yui no se recoupe pas forcément avec la forme est énoncéc de maniere indirecte. Cela exige un passage de l'expression implicite a la verifable intention du locuteur, comme dans la parabole Deus noire's me fon pas une blan'he. ce qui veut dire: Une forute d' antrme n'ercuse pase la mirré

Dans les deux cas, celui qui entend, contrairement a celui qui parle, procede par la logique. Face a cet wickal» logique de l'auditeur, toute information qu'on ess obligí d'extraire logiquement je dirai mime prwhologiquement - au cours d une commu nication verhalc. est, donc. consideréc sommo *autrement-dite*. Ainsi, l'autrement-dit, c'est ce qui est dit autrement par rapport au proprementdit qui, du fait d'être complet et explicite, dispense l'interlocuteur de combler les lacunes du locuteur pour mieux comprendre son dit. Iit commic toute langue se baracterise par la forme et le fond, on peu conclure que l'autrement-dit, c'est l'incomplet du côté de la forme et l'implicite du côté du fond

\section{L'AUTREMENT-DIT :}

\section{PRINCIPE DES I.ANGIUES}

Quand $\mathrm{ge}$ dis clangues". jentends, avant tout. de lingues naturelles, dont le comportement foncic rement irrationnel anticipe l'idee philosophique moderne d"ant1-Rationality" (I ash 1987: 357). Une langue formelle, construite par l'intellect. s'inspore plutô d'un vieil icléal rattonnel, avancés it toutes fins pratiques par Leibniz (Opuscules 190)3: 280). Lecart entre les langues naturelles et la langue formelle doit êre variable. Actuellement, il est difficile de proposer des méthodes quantitatives pour mesurer l'autrement-di entendu comme un ecart entre une façon incompletc et implicite d'énoncer la pensé dans unc langue naturelle et son expression parfaste dans unc langue imagenaire, une langue ctalon absolu.

(ependant, il est possible de détermuner le degre de manifestation de l'autrement-dit d une langue naturelle, choisic comme langue de reference. comme éalon relatif. J'ai tente anust dévaluer les moyens de "dire autrement". de l'unc des langues les plus alls gariques du monde. le français. face au quasi parallélisme entre ke logique et le verhal, caractéristique du russe.

Toute langue n'est qu une allusion. l'autrement-dit en est un vehacule indispensable D'apres ce paramète, on peut situer, par extemple. l'anglaıs entre le français et l'allemand el l'allemand, entre l'anglats el le russe. (ompares les termess, ne serait-ce yue d'apris le critère métrique qui veut yue plus il y at de consonnes, dites porteuse's de sems, plus c'est explicite: haut rendeme'nt, he'avy duts, grossis
Leistungsfähigkett, apparat bol'choj moch'nosti. Seule qui change, at fond. lorsquion passe d'une langue à l'autre, c'est la proportion de l'autrement-dit.

Or, ramener les diffierences entre langues a une unité graduée --- et c'étaut la l'objectif de ma seconde these consacres, tout comme ma premiere, a l'autrement-dit (Lobatchev 1987: 1992: 149.5) -. cest essayer de contribuer a la théric ett a la pratique de la traduction. Fi comme "langues" et "unite graduée"se rattachent a des domaines voisios, mais distincls, de la linguistıuec et de la psychologie, je fonde par là même une science autre que la psycholinguistique : une alinguo-psychologie.. Qui dit "l autrement-dit". dit "linguo-psychologie". Sans aucune contrainke, car at cour de la linguo-peychologie, on retrouve le' méme' ressort : l'autrement-dit.

\section{I.A LING:UO-PSYCHOLOCIE: DE: \\ IAALTREMENT-DIT}

Puisque des traits de la nouvelle science sont lies. pour reprendre le mot de Monique C. Cormier (1991 : 440). a "une ventilation des difficultés" préexistanles, notons-en quelques une's. Prenierement, il y a rupture entre la psycholinguistique et la linguistique. l'une étant centrée sur le psychisme, trop intériorisé, de l'èlre humain, l'autre, sur le systeme purement extérieur el trop deshumanise. de la langue.

Dơxiemément. contrairement a la psycholinguistique. la linguo-psychologic que je propose n'éloigne pas la linguistique du matériau de la langue, mais le maintent terujours atu premier plan. On voit bien que c'est plus qu'une simple querelle de termes. In effet, si la psycholinguistique ne s'ixcupe que de quelques spésificites dans la condulte langagiere, la linguos-psychologite, comme $x$ lentends, "attache a degager dans l'ensemble du discours et finalement danx la langue une caracléristicue profonde yui traverse devethmen ell des malloms.

In d'autres ermes, les differences yuantitatives entro la lan-ga-gi-sa-tion de la psychologie, réalisé en psycholınguistiquc. et la psy-cho-lo-gi-sa-tion de la langue, réalises en linguo-psychologice, peuvent etre compareses a la difference entre la petite partic de liceberg. partic vasible. et la monlagne de glace qui forme sil plus grande partec, invisible.

Et troisiemement. les differences qualitatives entre ces deux sciences se ramenent a la différence qui oppose des manifestatıons isolés du psychisme dans le discours à une representation unifice du langigge. comprenant allss bicn le mode objectut de sil perception - lautrement-dit que le mode ubjectif de sa création l'autrement-dire

Fatuc de place. nous ne peruvons que mentionner cette notion yui est sous-jatente à latutre. Fi comme laatrement dire fiera l'ohjet de recherches speciales. concentrons-nous ici sur celle ale l'autrement-dit.

On pourra le mieux examiner ce phénomenc à lexemple des risques que court la traduction suivant les recommandations de la peycholingustigue ou encore celle de la linguistique traditionnelle. Mettons 
que nous sommes en presence de deux personnages. dont l'un est artitraire et l'autre, résolu. Mais attention: l'un est Français el l'autre, Russe, ou vice-versa.

Eh bien, pour la psycholinguistique, qui n'est pas, linguistiquement parlant, une science comparative. la difference est trop minime pour qu'on puisse la prendre en consideration. Pour la linguistique traditionnelle aussi. Son echelle d'abstraction se trouve. face à cela, trop grande. On peut, bien sûr, comme le faisail Charles Bally (1951: 292), ranger les mots el groupes de mots parmi les moyens "directs" d'expression et l'intonation, l'ellipse et des procédés de syntaxe, parmi les moyens aindirectsn. Mais tous les moyens d'expression directs ne relevent pas du lexique. et lous les moyens indirects ne sont pas contenus dans la syntaxe.

C'est là une réalité dérangeante, un peu à la manière de notre autrement-dit yui en est la cause. Aussi se laisse-t-elle démontrer d'une façon plus naturelle a travers la comparaison des langues. La premiere surprise est qu'en russe. langue plutoò proprement-dite, une formule officielle d'interdiction $P$ ', gazonum no hodit' (litteralement: "Ne pas marcher sur les gazons») releve justement de la syntaxe, alors qu'en français. une formule analogue (Pelouse interdite/ repose sur le lexique. Et si on tient compte non pas de la marque formelle, mais, comme le veut le principe de l'autrement-dit, du contenu récl de l'énoncé. on voil ceci : la formule syntaxique russe relève de l'expression directe de la pensée, la formule lexicale française, de l'expression indirecte. Ai-je prouvé la le contraire de ce qu'avançait Bally?

Je ne crois pas. Il se trouve tout simplement que le fait - très abstrait en soi - que des moyens langagiers, utilisés dans ces formules, se rattachent a tel ou tel rang de la langue est impertinent. Dans d'autres cas, il est franchement secondaire. Or, ce qui compte ici en premier lieu est le fait de reciprocité : le russe est toujours plus proprement-dil que le français. le français est toujours plus autrement-dit que le russe.

Ainsi. le Russe dira à travers ses panneaux Ostorojno, zlaja sribaha (litteralement: Altention. chien méchont, alors que le Français ne dira que Atrention aus chien ou Chien méchant. Et comme s'il voulait nous montrer aussi un autre volet de l'autrement-dit qui joue non seulement avec l'incomplet, mais aussi avec l'implicite, il dira plus pour dire moins: Prenez garde au chien : Je mone la garde: Vous penére dans crlle conceinte a vos risques et périls. etc.

Pour en revenir a nos deux personnages, notons que traduire d'après les recettes de Bally ne resout le problème que partiellement. Eı encore, d'une façon très formelle. laissant de côté le gros de la question sur le direct el l'indirect et leur intermittence normative dans ielle ou telle langue. Sans cela, un homme simplement résolu, qui, avec la franchise des russophones, appelle les choses par leur nom, risque d'apparaitre aux yeux des Français comme un grossier personnage. Et, a l'inverse, une personnalite effectivement autoritaire et méme grossière qui. conformément aux règles du français, envelopperait sa pensée dans une forme plus ou moins détournée. pourrait passer, parmi les Russes, pour un homme simplement résolu.

Comment faire pour artêter dans les traductions. allant dans les deux sens. ce glissement de part et d'autre qui va jusqu à compromettre la validité du travail effectué ? La réponse me paraît claire: s'en tenir à une norme nationale, définie dans la linguopsychologic comparé commc un degré de l'autrement-dit, variant d'une langue à l'autre.

LAUTREMENT-DIT ET LA TRADUCTION : EFFETS INDIRECTS

Lexistence de l'autrement-dit, et aussi son importance pour la langue, sont attestees, quoique de manière indirecte, mais précise, par la pratique de la traduction et de l'interprétariat, où l'on voit les traducteurs et les interpretes préférer certains procédés à d'autres.

C'est ainsi que, dans la traduction orale consécu tive, l'interprète utilise non pas la sténographie qui note les mots eux-mémes, mais un système particulier permettant de noter les idées. Car a partir d'elles, des idées, il est plus facile de passer a l'autre langue qui privilégie d'autres aspects de la réalité que ceux retenus par la langue source.

On pourrait penser que cela ne s'applique qu'a la traduction orale, que l'on peut considerer comme secondaire. Mais pour les traductions écrites aussi et méme pour les traductions littéraires, il est parfois conscille, dans certains cas difficiles, de cacher avec la main la phrase en question, afin de se détacher des formes concrètes de la langue source et de passer ainsi plus librement vers les autres formes concrètes de la langue cible.

Lorsque l'interprétation consécutive se déroule dans le cadre de renconires "au sommet", de rencontres entre gouvernements. un interprete travaille de part et dautre : chacun traduit non pas a partir de la langue ectrangere vers la langue matemelle. ce qui paraitrait plus simple, mais de sa langue maternelle vers la langue étrangère parce que, ainsi. la traduction est plus sûre. On place donc la fidélité et la fiabilité de la perception au-dessus de la beauté de style. on sacrifie parfois même la correction linguistique la plus élémentaire.

Les interprètes en simultané affiment, en outre. qu'il est plus facile d'interpréter non pas à partir de la langue etrangere, surtout s'il s'agit du français, vers la langue matemelle, surtout s'il s'agit du russe, mais au coniraire. de la langue maternelle vers la langue étrangere. C'est que, subjectivement, la langue étrangère apparaît toujours plus autrement-dite que la langue matemelle. Et, de manière générale. il est plus facile de traduire a partir d'une langue à expressivité élevée vers une langue à expressivité moins élevée.

Ainsi, tous ces effets. aussi fins qu'ils soient, sont bien connus par les praticiens. Enfin. "connuss n'est. 
peut-être, pas le mol: plus senties, certainement, que connues. Pourtant. ils peuvent toujours être consideres comme une preuve de la quasi-impossibilité d'ignorer à la longue, la présence de l'autrement-dit. Justemen parce qu elle est parout. Confusément présente. mais partoul.

Cela fait penser au paradoxe nietzschéen de la connaissance. Lorsqu on essaic de regarder le miroir tel qu'il est. on ne découvre en lui que des choses qui s'y refletent. Si l'on veut attemdre le sens des choses, on tend vers cux la main et on touche le vide da muroir. Le phenomene de l'autrement-dit devient presque palpable de' qu'il depasse le cadre trickement lingusstique et reste insaisissible tant qu' il s' y replic.

Tout celil pour dire que les effets directs de l'autrement-dit sont plus difficiles a reconnaitre et qu'alors les traducteurs et les interpretes pourraien avoir besoin daide. En fatt, je $n$ 'ai pas grand-chose a leur offrir, sinon un quar de siècle d'observations et de recherches. Dans la partie sur la linguo-psychologie. il y en a déjà quelques-unes. En voici d'autres.

\section{L'AUTREMENT-DIT ET LA TRADUCTION : FFFETS DIRFCTS}

\section{Provenant de la linguistique générale}

Traducteur d'après la formation, je n'exuminais, pour construire ma typologie, que des rangs de langue présentant l'intérêt pour la traduction. Comme lors du passage d'une langue a l'autre on a surtout affaire a des mots et a des phrases. j"étudiais le lexique et lia syntaxe. Normalement, les typologues n aiment pas a les envisager ensenthle. les résultats étant beaucoup trop déroutants. Fi on est. généralement. incapable d'en tirer autre chose qu une vision gastronomique "La langue. c'est comme un gâteau, yuoi?... a plusicurs étagess.

Mais, primo, comparaison n'est pas raison. Sercundo. les traducteurs n'ont rien a faire de la typologie qui avoue son impuissance. Ce qui provoque le plus souvent l'échec des typologues est la contradiction qu'on observe dans les langues analytiques en général, et en français en particulier. Il y a, comme vous saves deux lendances, l'unce a se confiner surtout it un mot-racinc, ce yui suppose un raccourcissement. et l'autre à séparer au maxımum des unites langagières, ce qui suppose un rallongement. On semble etre dans l'impasse.

Or. cle qui peut nous tirer d'embarras est ke concep de l'autrement-dit. Pour celat, il faut que ses eléments constltuants se recoupent, ne serait-ce quen partie. avec les eléments de la typologic fornelle. kn effet. isolation et raccourcissement = l'incomplet. Mas. surtout. il faut qu avec leurs référencess, les premiers transgressent les reconds par rappont au fond. L.'incomplet, fiace a lit forme. est aussi incomplet face au comtenu. L'implicite, ce n est pas seulement une référence ì lat séparabilité formelle des constituants et au raliongement, mais, avant tout. une référence par rappert au fond. Finalement. cest ce croisement du fond et de la forme qui permet de voir au-delà du mouvement capricieux de la forme, qui se raccourcit a un niveau pour se rallonger à un autre, une logique suprême du fond: maintenir partout dans la langue un seul et mème degré de l'autrement-dit. On retrouve par la mème une cohérence perdue de la langue.

Ainsi, on réalise qu'une réduction de l'énoncé jusqu'à un mot (par exemple. line allume'te' au lieu de lioss ase une allumeste" ") aussi bien que son śtirement jusqu il contient. en plus. unc periphrase

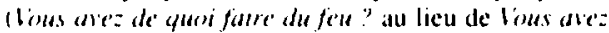
dis fels.") ouvre egalement une perspective pour une variation du sens. Il est vral qu en l'absence du con. texte. ou de "l'assertion", comme dirait Peirce? "toute proposition n devient floue. Mais la fluctuation du sens doit être particulièrement grande. lorsquion secearte du juste milieu de la forme, et peu importe alors la direction qu' on prend. Hors la situation. avec line allumette" $\therefore$ on reste dans le vague : " $"$ 'st a domner ou d prendre" comme on ne voit pas trè bien avec loms arez de quoi faire du fe'u? si c'est au sen. figuré ou au sens propre que la périphrase est employée et si c'est au sens propre, s'agirait-il d'un briquet ou d'ume allumeste? Il n'y a pas vraiment moyen d"echapper a l'autrement-dit, quelle que soit la solution que vous offre le langage sur son chemin : prendre un raccourci ou faire un détour?

\section{Effets directs provenant de}

la linguistique comparée

Je vous fais grâce ici des détails de mon analyse contrastive des langues yui comprend des dizaines et des dizaines de parametres lels que corpus des premiers mille mots les plus usuels, leur fréquence relative. dimension du vecabulaire a travers les dictionnatires fréquentiels, ensemble des moyens phrasésologiques, polysémie et homonymie, synonymie et paronymie, distribution des modes de expression indirect el direct dans les textes. etc. Fi, detrompez-vous. l'ordre aindirect et direct. n 'est pas un kapsias lingsude, au contraire. c'est bien voulu de ma parn pour souligner yue lautrement-dit lemporte partout sur le "proprement". En plus, ici, il inporte aussi de savoir comment cela se passe dans les langues comparées? Ith bien, à en juger d'apress tous ces parametres, le français devance le russe dans sa progression vers l'autrement-dit, et cela avec unc étonnante régularité qui se chiffre dans un rapport de 3 it 1 : le volume émantique du mot trançass est trois fois plus grand. le nombre d'homonymes aussi. la quantite de péruphrases et ainsi de suite.

Dans l'ensemble, on pourrail tigurer ce rappon entre le français et le russe par une analogie. Imaginey que le Ruse et le Français empruntent la méme route. its aturaient alors affaire à des messages differents. Si l'un ne recoit que les interdictions $1 N^{\prime}$ hoddir'. Ne

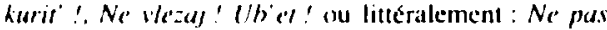
marrher! Ne pas fumer! Ne erimpe pas! (a) ra te tuer' '). l'autre ent plutô lobjet d'avertissements

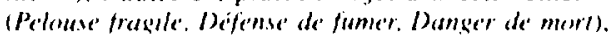


l'objel de sollicitations (Priere d'observer le silence. Priere d'attendre ici. Priere de patienter) ou meme l'objel d'encouragements (Merci de vous abstenir de fumer: Merci de céder votre place. Merci de ne pas obstruer les portes). Ce qui se trouve ici en jeu outre les différences culturelles évidentes. outre la distinction, qui vient immédiatement à l'esprit, entre des regimes dictatoriaux et des regimes démocratiques - c'est avant tout, tout à fait dans la mesure où la langue influence le resie'. Ies degrés différents de l'autrement-dit qui font deux normes nationales distinctes.

\section{L'impact direct pour la traduction}

Qui parmi nous ne s'est pas laissé dire un jour que, dans la traduction, tout était une question du goa et qu'on pouvait traduire comme ca ou comme ça, ou encore comme ça? Qui n'a pas révé après cela d'avoir des repères sors. plus palpables ? Eh bien, une vieille differenciation entre l'obligatolre el le facultatif, que je trouvais toujours artificielle, tombe. Tout devient obligatoire du point de vue de ce degre unique de l'autrement-dit a l'intérieur d'une langue qui en a des limites superieure et inférieure. Vu la mobilité de la norme entre ces limites, sa definition plus precise (cj. celle de la partic sur la linguo-psychologie) sera la suivante. La norme nationale est le degré maximal et minimal de manifestation de l'autrement-dit, variable d'une langue à l'autre (voyez la figure)

limite supérieure de l'autrement-dis

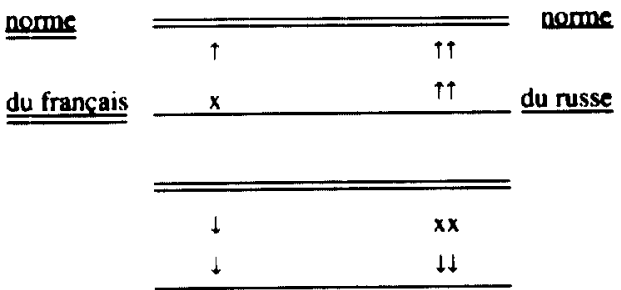

limise inferieure de l' autrement-dit Figure 1

Pour rester dans le figuratif, je vais m'appuyer sur lexpérience de tous, puisque tout le monde est censé. une fois dans sa vie, avoir écouté a la radio ou regardé a la télevision un match de hockey. Je vais donc emprunter mes exemples aux commentateurs sportifs, si eloyuents et persuasifs qu'ils nous renvoient toujours une image fidèle de notre langue. Mais avant de venir à cette expérience positive. commençons, pour ne rien omeltre, par une pratique negative, celle qui est à éviter.

On voit ainsi que les transgressions de la norme (marquees sur la figure par les $x$ barrant les fleches $\downarrow \downarrow$ et $\uparrow$ ) sont, quand on traduit en français (souliene deex fois et fleche double $\downarrow \downarrow$ ), une intensification excessive : C'est le point le plus faible dans son jeu - depassement par le bas du degré minimal d'autrement-dit admis dans cette langue - et, en russe (souliené une fois et fleche simple $\uparrow$ ). au contraire, une trop grande attenuation de la penste : Eto ne lutchee $v$ ego igre (litteralement: Ce n'est pas ce qu'il y de meilleur dans son jeu) - depassement par le haut de son degré maximal d'autrement-dit.

Observer la norme nationale, $c^{\circ} e s t$ comme chanter un hymne $d$. Suivre. a la figure. les fleches non barté $\downarrow$ et ${ }^{\uparrow \uparrow}$, c'est aussi en quelque sorte savoir chanter la chanson. Aussi allons-nous ajouter un autre exemple, traitant le principe de la musique ( $f f$. l'introduction), qui se montre, par ailleurs, aussi souple que celui de la parole.

Alors. pour faire de bonnes traductions à partir du français, il ne faut pas oublier que la limite superieure de l'autrement-dit en russe est plus basse et donc renforcer l'expression. en explicitant et en ajoutant aussi des explications ( $(\downarrow)$ : Eto naibolec slaboe mesto " ego igre (litteralement: C'est le point le plus faible dans son jeu): Sut' v tone, a ne v slovah (litteralement : L'essentiel. c'est l'air, el non les paroles).

Mais lorsqu' on traduit du russe, il s'agit, au contraire, de tenir compte de la position relativement elevee en français de sa limite infericure de l'autrement-dit et donc attenuer, en «implicitant" el en retranchant $(\uparrow \uparrow)$ : Ce n'est pas ce qu'il y a de meilleur dans son jeu. C"est l'air qui fair la chanson.

\section{CONCLUSION}

Avec une citation de Wilhelm von Humboldt. pieine de signification pour moi et dont la traduction un peu erronte n'est. peut-êre. pas sans intérêt pour vous, je crois avoir trouvé un moyen idéal, concret el abstrait en même temps, de conclure. "Comme l'homme est par nature, universellement, predisposé au langage, et que tous les hommes doivent porter au fond d'cux-mémes la clef qui donne l'intelligence de toutes les langues. il s'ensuit qu'elles doivent toutes impliquer une forme fondamentalement identique.... (1974: 404).

Très important ici est le demier mot. L'original allemand (1880) dit : "gleich" ce qui veut dire égal en arithmétique, peut-etre, mais, en dehors des evidences du type «deux et deux font quatre». plutôt similaire ${ }^{4}$. Le traducteur a donc le choix entre un terme étroit qui rend le texte rigide (raison pour laquelle identique clôt la liste des variantes proposées par le grand dictionnaire de Grappin (1992) et un terme large qui rend le sens souple (raison pour laquelle, en partant de similaire, on arrive à gleichartig dans l'espace méme du petit dictionnaire de Villain (1964). Que fait le traducteur? ll opte pour identique - erreur que commettent aussi d'autres traducteurs qui ont plus d'excuses du fait de ne pas être aussi bien placés par la langue cible (pour le russe, voir Humbold 1984 : 227) - ce qui l'oblige. quelques lignes après, a faire un renvoi pour la note: “Renversement, ou du moins correction des enonces du chap. $35 m(1974: 420)$. 
Or, que ferait un autre traducteur, celui qui serait du courant de la linguo-psychologie, ou de notre theorie de l'autrement-dit? Il se dirait que, face a l'allemand. le français devrait se comporter à peu près de la même manière qu'il le fait dans le cas du russe (voir la fig. 1). Fit comme le degré de l'autrement-dit en traduction française dépasse celui qu'accuse l'original allemand, il faut que le terme choisi son auss moins engageant pour le traducteur que possible. Sans parler de l'économie de toute une note dont je n'al reproxluit ici qu'une petite partic et dont l'ensemble. d'ailleurs, me donne raison), le terme approprié (ce sera le mot similaire), celui qui traduit le rapport des langues, rend aussi le sens de l'énoncé.

En effet, cette difference, apparemment infime. est en réalité de taille. Et Humboldt lui-même en est parfaitement conscient, puisqu il cherche à préciser sal pensée dans une phrase qui suit. pratiquement. celle que nous avons cité avant, sous une forme traduite. Pour plus de sécurité, la suite sera reproduite dans sa forme originale. «Sie (Verschiedenheit) ist aber - ce "mais" noppose ici que dissemblance et ressemblance - mannigfaltig in den Sprachen vorhanden. und nicht nur in den blossen lauten, so dass dieselben Dinge anders beesechnet würden"... (1880): 3(17). (ce qui veut dire: les différences entre les langues dépassent leur côté phonétique et vont hien au-delà de la façon de désigner les choses autrement... Sans cette dissemblance intérieure des langues, lout serail fige. et il suffirait, selon l'idé chomskienne, de réduire les structures superficielles des langues a leurs structures profondes, pour acceder au point mon, is une paix absolue

Heureusement. ce n'est pas cela, mais le contraire que nous apprend la pratique de la traduction. Elle nous apprend egalement qu'aussi loin yu'on puisse remonter dans le temps, on ne trouve pas de rupture significative de la tradition. Il n'y avait ni d'avant, ni d'apres Babel. En effet, le monde est, el a toujours

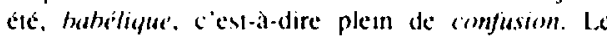
proncipe originel du langage ne peut etre autre chose que le principe universel des langues: l'autrementdit yut est al la fois dissemblance et ressemblance. Constamment mise devant te choix entre l'identique ou le similaire. la traduction optait presque toujours intuitivement il est vrai, or il faut que ce soit consciemment - pour plus de souplesse.

Se faire comfondre a looriginal ne fut-il pas une de ses plus grandes ambitions?' Fi je l'approuve. En périphrasant Humboldt qui disail que toute compres. hension ext une incomprehension. je dirais qu'il est imporsible de comprendre sans confondre. Je dirais même que confusion la constitue un élément indis. pensable de la compréhension. Vous, moi. vetre auteur, mes lecteurs - toul ça se déplace dans l'interaction et se confend sirns jamais fondre dans l'identique. Et c'est bien comme cela, car entre l'identique el le stmilaire, il y a un menterstice nécensaire pour créer de lespace a l'autrement-dit. (et espace est aussi vital pour la traduction que l'autrement-dit qui en est le principe.

\section{BORIS LOBATCHEV}

Momeréal, Canada

\section{Notes}

1. Quelques remarques préliminaires sur le style de notre article. "Phénoménocentrique". il diffère de la plupart des ouvrages qui sont "objectocentriquess, par son côte explicatif plutot que descriptif. D'vù la necessite de la synthese: le va-et-vient entre les parties est indispensable pour mieux travailler l'idée de l'ensemble.

2. Cité d'apress Fouchier-Axelsen Berthe (1992): "Fragments d'un discours linguistique (ou Peirce et le langage'm, Langues of linguisucuer, 18, p. 197.

3. Pour réaliser combien cette idée est présente dans l'esprit humain, il suffit de se référer. par exemple. a deux grands linguistes ou, si l'on veut. deux sémioticiens: Humboldt et Peirce. Espace l'un de l'autre par le temps, ils convergent dans ce point. le premier consacre l'ouvre de sa vie à létude de linfluence de la langue sur le développenent intellectuel: "Weber die Verschiedenheit des menschlichen Sprachbaues und ihren I:influss auf die geistige Entwicklung des Menschengeschlechtsw (18.30-18.35). Le deuxieme Eerit en 1903 : all est fatux de dire simplement qu'un bon langage est imprertant pour bien penser : car il est lessence même de la pensece foile d'apres Fouchier-Axelsen $1992: 2011$.

4. La notion de lidentique appartient aux sciences dites exactes. Depuis Aristote. on n'identifie que des objets dont le nombre est un. Il s'ensuit que le similaire, lui, ne se laisse étahlir qu'entre les objes dont le nombre égale ou dépasse deux. comme on $k$ remarque aussi, en se posant la question usu bien, ou bien?". le similaire est passager. l'identique est constant. le similaire est gradue, l'identique ne lest pas. ( $f$. Nina Arutjunova (1983) : "Tojdestvo ili podohie ?" [Identite ou similitude ?. Prohlemy strukturmo) lingurstiki 1981. Moscou. Nauka [Science]. pp. 3-22. Le similaire implique le non-identique. je dirais mème : ressemblance implique nonressemblance el dissemblance. En ralfongeant l'échelle, nous levons la frontiere similaire/ identique qui oppose les langues particulières et te langage humain, ou. dans la terminologie d'Humboldt, les formes el la forme. Pour aller de la dissemblance (langues) a la ressemblance (langage), in in'a pas hesoin de quitter le domaine du similare. Surtout quand on decouvre à deux niveaux le méne principe d'autrement-dit qui l'incarne : variance et graduation. caractéristiques constantes des scicinces humaines. Lidentique commence à un niveau supérieur. non au niveau des objets purs, non au niveau mixte des 
objets (langues) et des phénomènes (langage). mais au niveau des phénomènes purs d'où toute comparaison est exclue et où seule la distinction entre les phénomènes est possible. L'exemple de l'identité qui s'impose. c'est l'autrement-. a l'oppose du proprement-dit. wLe caractère de ce qui est un», dans la définition de l'identité par Roben, veut que le vrai principe se distingue du faux jusqu à éliminer ce dernier. Donc, pas de proprement-dit.

\section{REFERENCES}

BALLY, Charles (1951): Traite de stylistique fransaise. Paris, Klincksieck.

BARTHES, Roland (1984): Essais cririques IV the hruissement de la langue. Paris, Éditions du Seuil.

BREAL. Michel (1897) : Essai de sémantique (science des significationsi, $1^{\text {re }}$ ed. . $4^{c}$ ed. 1908, Paris. Hachette.

CORMIER. Monique C. (1991) : :Traduction de texies destines à des specialistes : approche pédagogiquew. Meta, 36-2/3, pp. 440-447.

GOUADEC, Daniel (1990): "Traduction signalitique. Meta, 35-2, pp. 3.32-341

GRAPPIN, Pierre (1992): Dictionnaire franşaisallemand / Deutsch-franzisisches Wörterhuch, Paris. Larousse. $780+780 p$.

HUMBOLDT. Wilhelm von (1880): Über die Verschiedenheil des menschlichen Sprachhaues mit erläuternden Anmerkungen und Excursen sowie als Einleitung : Wilhelm von Humboldr und die Sprachwissenschaft. herausgegehen und erläuterf von August Friedrich Pott. 2 Bände in 1 Band. Nachdruck der Ausgabe Berlin 1880 . Documenta semiotica, Herausgegeben von Walter A. Koch. Serie 1. Linguistik, 1974. New York. Georg OIms Verlag.

HUMBOLDT, Wilhelm von (1974) : Introduction d I'cruvre sur le kavi et d' autres essais, traduction et introduction de Pierre Caussat, Paris, Seuil.
HUMBOLDT, Wilhelm von (1984) : Iabrannye trudy po jazykoznaniu [Euvres choisies de linguistique], Moscou. Progress.

LASH, Scolt (1987): "Modernity or Modernism?". Max Weber, Rationality and Modernity, London, Allen \& Unwin, pp. 355-377.

LEIBNIZ. Wilhelm Gottfried (1903) : wLingua rationalis». Opuscules ef fragments inedits, par Louis Cuturat, Nachdruck der Ausgabe Paris 1903: Hildesheim, 1961, Georg Olms Verlagsbuchhandlung, S. 280.

LOBATCHEV. Boris (1987): Sopostavlenie francuskngo i russkogo jazykov na osnote javlenja inoskazarel' nosti / Confromtation du fransais ef du russe d iravers [' autrement-dit]. These de doctorat en traductologie. Moscou.

LOBATCHEV, Boris (1992): L'autrement-dit, concept central de la linguistique comparde permettant de ramener les différences entre langues d une unite graduce. These de doctorat en linguistique, Paris.

LOBATCHEV, Boris (1995) : L'autrement-dit. Paris. L.Harmattan, coll. «Sémantiques», $158 \mathrm{p}$

NOUSS, Alexis (1990) : «Babel : avant, apres», TTR. 3-2, pp. 53-70.

NOUSS. Alexis (1993) : «De la possibilite de traduire la potsie : la theorie de la traduction d'Hermann Brochn, $6^{e}$ Congres annuel de l'Association canadienne de traductologie, Carleton, le 1-3 juin. Bulletin de l'Association, pp. 6-7.

PEIRCE, Charles Sanders (1977): Semiotics and Significs, Bloomington, Indiana University Press.

PIRONI. Michel el Jacques ROUX (1993) : Babel ou le mysiere des langues, cuvre dramatique. ART'M en collaboration avec Les Cahiers de l'Égaré.

VILLAIN. Pierte (1964): Dictionnaire allemandfransais, fransais-allemand. Paris. GarnierFlammarion, $460 \mathrm{p}$ 